\title{
Prevention is better than cure
}

\author{
Leszek Borysiewicz
}

\begin{abstract}
The history of the control of infectious disease reflects the balance between our understanding of the nature of infection, the mechanisms of host resistance and the available technology to deliver an intervention. However, behind this 'biomedical' construct lies a deeper set of issues which embody serendipity, public understanding, and population-based intervention and its acceptability by the wider community, often complicated by the decisions and subsequent vacillations of policy makers.
\end{abstract}

\section{A tale of two vaccines}

In 1796 a remarkable clinical experiment took place in the village of Berkley in Gloucestershire. Since that time its story has been recounted, and embellished on numerous occasions, so it is as well to go back to Jenner in the original source ${ }^{1,2}$ :

The more accurately to observe the progress of the infection, I selected a health boy about eight years old for the purpose of inoculation with the cowpox. The matter was taken from the suppurated sore on the hand of a dairy Maid who was infected by her master's Cows, and it was inserted on the 14 May 1796 into the arms of the Boy by means of two superficial incisions each about three quarters of an inch long. During the whole of [the ninth day after this] he was perceptibly indisposed and had a restless night; but, on the following day he was perfectly well.

We are left to imagine the impact of this on Jenner. However, he proceeded to complete the experiment:

On the 1st of July following this Boy was inoculated with Matter immediately taken from a smallpox Pustule. Several punctures and slight incisions were made in both his arms, and the matter was well rubb'd into them, but no disease followed.

The consent of the subject, parents and ethical approval is left as a matter for conjecture. There was and remains some controversy as to how good a clinical investigator Jenner was. ${ }^{3}$ His treatise describes observations of pustules on the nipples of cows and their transmission to 'domeftics employed in milking. He describes a systemic illness that followed this exposure. However, he also postulated the transmission of this disorder from horses to cows, which was entirely erroneous. ${ }^{3} \mathrm{He}$

The Harveian Oration is delivered annually at the Royal College of Physicians of London under an indenture of William Harvey in 1656. This article is based on the 2009 oration delivered on 20 October 2009 by Sir Leszek Borysiewicz, chief executive, Medical

Research Council, London observed several cases of protection from smallpox after exposure to cowpox, inoculated smallpox into those who were exposed to cowpox, and finally undertook the experimental vaccination and challenge of James Phipps.

Jenner repeated this study on several occasions before publishing 'An Inquiry into the causes and effects of the Variolae Vaccinae, a disease discovered in some of the Western Counties of England, particularly Gloucestershire, and known by the name of the Cow Pox'. This was not the first attempt at publication as he had previously submitted this to the Philosophical Transactions of the Royal Society. He was mortified when the manuscript was rejected as the 'evidence he had adduced was considered to be insufficiently conclusive to justify his claims for the prophylactic power of cowpox'.

It is important for us to recognise the context in which these studies were performed. Smallpox had been a scourge of mankind since earliest times; probably originating in the Far East, recognised in Baghdad with a possibility that it may have afflicted Ramses V in ancient Egypt. ${ }^{5}$ However, epidemics were well recognised and after the plague, it was the most feared contagion. There is circumstantial evidence that the disease had previously had a lower mortality rate, but it has been suggested that, exacerbated by industrialisation and urbanisation in the late 18 th century, ${ }^{2}$ mortality had reached approximately one death in six or seven attacks. ${ }^{5}$ In 1796, there were 3,500 deaths recorded from smallpox in London. No cure was available although 'quack' remedies abounded that often did more harm than good. ${ }^{6}$ It was recognised that contact with a case may result in milder disease leading to the practice of variolation promoted by Lady Mary Wortley Montagu during the epidemic of 1721. This was publicised and tested (on prisoners in Newgate who were promised reprieves for submitting to the 'experiment') by Dr John Woodward. However, by 1728 it was noted that this procedure was variable, could promote spread of the disease and had a mortality of $2-3 \%$. 2,4

Remarkable as the original discovery was, the real interest in the context of this presentation is how the procedure found acceptability under the prevailing conditions. At first, vaccination was criticised and derided for many years. This changed quite rapidly, perhaps in no small measure due to the procedure finding favour with the royal family. This early recognition of the importance of vaccination as a procedure is reflected in the awards made to Jenner. In the UK, two awards were made. Firstly, a society (the Royal Jennerian Society) was established to provide vaccination to the poor - free of charge - with the King as its patron. This royal support probably played no small part in the award of a grant of $\mathfrak{1} 10,000$ by the House of Commons, so that 
Jenner should not be out of pocket because of his discovery and its promotion. ${ }^{4}$

Secondly, the Royal College of Physicians (RCP) was asked to opine on the novelty and value of vaccination in 1806. In an enquiry, the College supported the case and a further grant of $£ 20,000$ was made to Jenner. Parliament ordered the RCP to organise and superintend distribution of vaccine, which it did until 1861 . However, ultimately Jenner was disappointed by his interactions with the College as he applied for membership in 1814 but was informed that, despite his many international honours, he could not be a member without sitting the formal examination which required written papers in Latin and Greek. He decided that in his mid-sixties he would not undertake these additional studies. ${ }^{4}$

The proponents of vaccination immediately tried to push for its greater uptake, often citing the problems of competitive practices of established clinicians using variolation and (in Jenner's own words) ' $\ldots$ the caprices and prejudices of the misguided poor.' ${ }^{4}$ Proponents of greater uptake of vaccination echoed Jenner's sentiments and a range of literature was used in different cities in the UK to 'shame' people into cooperation.

Vaccination, although gaining popularity, was not without significant problems which are evident from the records:

- It was difficult to ensure a supply of vaccinia. This is a rare disease of cattle; therefore a continuous source of material was required. Different techniques were tried, notably armto-arm vaccination or drying plasma on to glass (less effective). The former method was adopted to transfer material by ship to North America in 1803 by order of the King of Spain. ${ }^{2}$ Jenner recognised how important it was that the material obtained from the cow or patient was in the 'vesicular' stage rather than 'pustular'. However, the dearth of material often resulted in supra-infection with cutaneous complications.

- There was no standardised procedure or training.

- There were no established criteria as to what constituted a 'successful' vaccination (thereby confounding data collection).

- Longevity of effectiveness. From its earliest use, effectiveness was often associated with recent vaccination and revaccination was introduced on a national scale in Germany.

- There was a sceptical public.

The practice of variolation was banned in 1840 , as it was seen as a means of transmitting smallpox rather than protecting from it. Vaccination won the support of many, such that by 1853 it was made compulsory for all infants under the age of four months. How effective was vaccination in these early years? In assessing this it should also be remembered that careful studies have shown that the incidence and severity of smallpox was falling regardless of vaccination..$^{5}$ Opinions differ, but some evidence supporting an impact of vaccination is forthcoming especially in studies of outbreaks. The key epidemic was between 1870 and 1873 when there were 44,079 deaths from smallpox in the UK (10,000 in London). ${ }^{2,5}$ While the high mortality suggested to some that vaccination was ineffective, ${ }^{3}$ observations to the local government board that the average mortality was $148 / 100,000$ in London compared with $400-500 / 100,000$ pre-vaccination. It was noted that vaccination uptake was $93.6 \%{ }^{2}$ Secondly, further data were available as a result of the Franco-Prussian war of 1870 , fought at the height of the European epidemic. There were 4,835 cases with 278 deaths in the German army where revaccination was compulsory, whereas there were 14,178 cases and 1,963 deaths among French (unvaccinated) prisoners of war alone. This was commented on by Richard Quain, the harveian orator in 1885, speaking on 'The history and progress of medicine ${ }^{77}$ as 'one fact is worth a ship-load of argument'. His 'facts' were:

- since 1874, 'not a single case of death from variola' was recorded in the German army as it 'dwells in the midst of a population protected by compulsory revaccination'

- Ireland as the 'best vaccinated country in the world' was 'practically free of smallpox'.

Despite the then harveian orator's protestations to the contrary, we are forced to conclude that the momentum for vaccination was driven by need and rhetoric rather than hard data as we know it today.

The 1853 Act to extend and make compulsory the Practice of Vaccination crystallised much of the opposition to vaccination. It is difficult to summarise the number of allegiances of various movements in Victorian Britain that found a focus in opposing vaccination. ${ }^{4}$ Their objections centred on four main arguments:

1 Bestiality - it was wrong to use animal material to treat humans in this way. This was perhaps the earliest opposition to vaccination and started soon after Jenner's studies were published. It found voice among the clergy and in the House of Lords in 1814. But it was largely countered by the introduction of arm-to-arm vaccination gradually fading as the century progressed, ${ }^{4}$ although it was not until 1896 that provision of vaccine was from animal sources alone. ${ }^{2}$

2 Disbelief in the effectiveness of vaccination - sometimes with an analysis of mortality data but sometimes directed at Jenner personally. ${ }^{3}$

3 Social reformers who contended that smallpox was not the underlying problem but merely a symptom of the poverty and degradation that pervaded Victorian cities.

4 Those opposed to the principle of compulsion or its direct effects on those caught by the draconian provisions of the 1853 act and its subsequent amendments. These made fines and even imprisonment cumulative and antivaccination societies were established in many parts of the UK. The discontent was made more widespread by loose alliances between those opposed to compulsion, principle-based opponents, medical opponents, antiestablishment organisations and individuals as well as numerous citizens. The medical hierarchy largely supported compulsion as evidenced by a letter to the president of the Board of Health by John Simon, an established figure in the public health movement and a strong supporter of the 1853 Act: 
It goes with the credulity which characterises the present age to be incredulous of proved truth. Alike in rejecting what is known and believing what is preposterous, the rights of private foolishness assert themselves. It is but the same impotence of judgement which shrinks from embracing what is real, and lavishes itself upon clouds of fiction. ${ }^{7}$

The proponents did not engage or understand these concerns and probably made little effort to do so. In contrast, those charged with implementing the law refrained from using it to prosecute individuals. In the end, the opposition to compulsion achieved its goals as the Poor Law Guardians, responsible for implementing vaccination throughout the country, alongside magistrates, refused to implement compulsory vaccination. The act was repealed in 1906.

As the vision of national smallpox control changed to one of global eradication, the key issues associated with implementation had to be addressed. The problems associated with effective vaccination required a transportable and effective vaccine which became available (at the end of the 19th century), delivery systems and processes were standardised and gradually improved. Above all once global eradication was the agreed goal the steadfast commitment of the World Health Organization (WHO) and its member governments, especially in the latter stages, was essential. In 1966, there were still 10-15 million cases of smallpox in 33 countries and the WHO voted to develop a 10-year campaign to achieve eradication. By 1976, the last cases in Bangladesh and Somalia were reported, but tragically the last fatal case occurred in Birmingham as the result of an accidental laboratory release. In 1980, the WHO declared the world free of smallpox. ${ }^{8,9}$

Smallpox has been held as the paradigm for eradication programmes and it has spawned other programmes, the most advanced being the WHO Poliomyelitis Eradication Programme. Much has been achieved towards this goal and eradication is now within grasp but the issue which bedevilled smallpox eradication, namely acceptability in some countries, particularly Northern Nigeria, is recurring. ${ }^{10}$

Can we draw common themes which led to the success of smallpox control and eradication? Key stages and associated issues were:

- biomedical observation and development of the concept as a technical achievement

- clinical experimentation to establish proof of principle

- availability of the intervention and scalability for population-wide application

- monitoring of impact

- extension to public policy

- opposition to the policy - this was inevitable and needed to be considered, anticipated and addressed

- public acceptance of the policy or its modification

- implementation and delivery of the policy

- vision of the ultimate goal.

While these were achieved with smallpox vaccination, it is interesting to draw a comparison with a modern-day implementation issue such as the combined measles, mumps and rubella
(MMR) vaccine. When MMR was first introduced in 1988, there was widespread acceptance of the vaccination. However, as has been well documented, this was undermined by the suggestion that the vaccine could be associated with autism and/or Crohn's disease. ${ }^{11}$ This prompted numerous studies and most accept that there is little evidence to support the proposition. Epidemiological studies have failed to detect any excess of cases of autism and virological studies have failed to confirm earlier observations. ${ }^{11-13}$ However, the damage was done in the UK which impacted more widely. ${ }^{14,15}$ The level of mistrust was fuelled for a variety of reasons:

- the highlighting of individual patient stories in the media

- a distrust of information, especially that provided by government and commercial sources ${ }^{11}$

- a general sense of anxiety even in those who had had their children vaccinated ${ }^{16}$

- $\quad$ information and views of professionals. ${ }^{11}$

The impact of the media is difficult to understate in this context. Indeed, in a recent analysis by Tom Whipple in The Times, it was recognised that:

For almost a decade, from 1997 to 2004, newspapers cheerfully spread the idea that the MMR vaccine caused autism. They trumpeted compelling anecdotes from teary mothers, made fun of those silly science boffins, and sold a lot of papers. It was fun. In 2004, something that they hadn't thought of happened: MMR uptake dropped to 80 per cent of two-year-olds from 92 per cent in 1997. Doctors - including one of those who had originally posited the autism link - warned that children could die. The game, it seemed, was interactive. ${ }^{17}$

Less than one third of news stories between January and September 2002 pointed to the overwhelming scientific evidence that MMR was safe, but continued to promote the anecdotal. ${ }^{18}$ Public opinions about MMR safety, documented by market research companies ICM and Ipsos MORI, suggested that the raised anxiety was associated with this reporting. ${ }^{14}$ However, Whipple also observed that even when the stories changed in tone regarding safety, this still impacted detrimentally on vaccination rates, which led him to conclude that:

The temptation for newspapers is to start writing about MMR again, encouraging vaccination. Perhaps, though, the best thing we can do about it is just shut up. ${ }^{17}$

It is easy to use the media as a convenient scapegoat but from the common issues that arose in the context of smallpox vaccination, the biomedical community forgot that opposition will occur in the context of any innovation. This message was further underlined in the Peckham Report, ${ }^{19}$ yet we must ask ourselves if enough attention was paid to this likelihood and was the concerted attempt to counter the proposed erroneous links sufficient? So this intervention has proved less successful than it should have been, not because of a failed intervention due to technical or delivery issues but because we have been unable to counter the opposition to it effectively and in a timely manner. This is an important lesson that must be learned and anticipated 
if the myriad of potential preventative interventions are to be effectively implemented in the future.

\section{The white death - tuberculosis}

Lord Byron: How pale I look! I should like, I think, to die of a consumption! Lord Sligo: Why of a consumption? Lord Byron: Because then all the women would say, 'See that poor Byron how interesting he looks in dying!' 20

It is difficult to overestimate the importance of tuberculosis (TB) during the 19th century. The major contagious diseases were still rife during the mid-century, but they were dwarfed as a cause of mortality by TB (Fig 1). A study of the Statistical Records for London (1847-50) indicates that approximately $16 \%$ of all deaths at all ages were attributed to TB. Yet it is also clear that TB was on the decline rather than on the increase. From less accurate records, the best estimates suggest that mortality reached its peak at the start of the 19th century. ${ }^{21}$ This decline in mortality is better documented for the latter part of the century (Fig 2a) and was observed in all parts of the world. ${ }^{22}$ It was also apparent that the mortality was higher in cities as compared with rural areas (Fig 2b). However, the reasons for this decline are less easy to understand. Favourite hypotheses include:

- improved socioeconomic conditions, in contrast to the pervading view of the impact of industrialisation in the 19th century

- improved nutrition consequent on the socioeconomic improvement

- application of rudimentary public health measures especially after the realisation of $\mathrm{TB}$ as an infectious disease (coupled with the Germ Theory of Disease). ${ }^{21}$

The decline in TB mortality continued into the 20th century to reach a nadir in the 1990s. Again some observations can be made from a scrutiny of these numbers. Firstly, in countries affected by the major conflicts of the 20th century, there has been an associated increase in TB mortality during the first and second world wars, suggesting that any breakdown of improved socioeconomic conditions enables the disease to reappear very rapidly. Secondly, during the last century, several attempts at prevention and control have been made and it remains difficult to be certain of their additional impact on the falling baseline of TB mortality:

- The Sanatorium Movement. Initially this started in Silesia but rapidly spread to other parts of the world, especially America. It potentially brought comfort and relief to sufferers but was it effective? This is unclear. Daniel cites a study of 4,000 patients in Lancashire in 1923 comparing those treated at home with those in sanatoria. ${ }^{23}$ Of sputum negative patients, $14 \%$ died if admitted (compared with $38 \%$ at home), but death rates rose to $61 \%$ and $81 \%$ respectively among sputum positive individuals. Studies from New York sanatoria showed similar results. However, whether sanatoria limited spread and acted as a prevention by isolation is less likely.

- Mass X-ray screening. This was initially introduced by the armed forces in the first world war and dominated as a major diagnostic and screening procedure for the first half of the 20th century. ${ }^{24}$ Unfortunately, diagnosis was not followed by effective treatment until the 1950s. It was modified by the introduction of mass miniature radiography for screening but results such as the 21,627 individuals screened in Wales resulting in a yield of $0.6 \%$ led to its abandonment. ${ }^{25}$

- BCG vaccination. BCG vaccination began in man in the 1920s following attenuation of the organism by serial passage. Initially there were several incidents where the degree of attenuation was questionable but by 1948 , UNICEF and the Danish Red Cross embarked on a programme of tuberculin testing and BCG vaccination with a
Fig 1. The impact of tuberculosis as seen in the average mortality from infectious diseases in London between 1847 and 1850 (data from the Royal Statistical Society). During this period (1851 census) the population of London was 2.6 million and the average all cause mortality was 16,300 per annum.

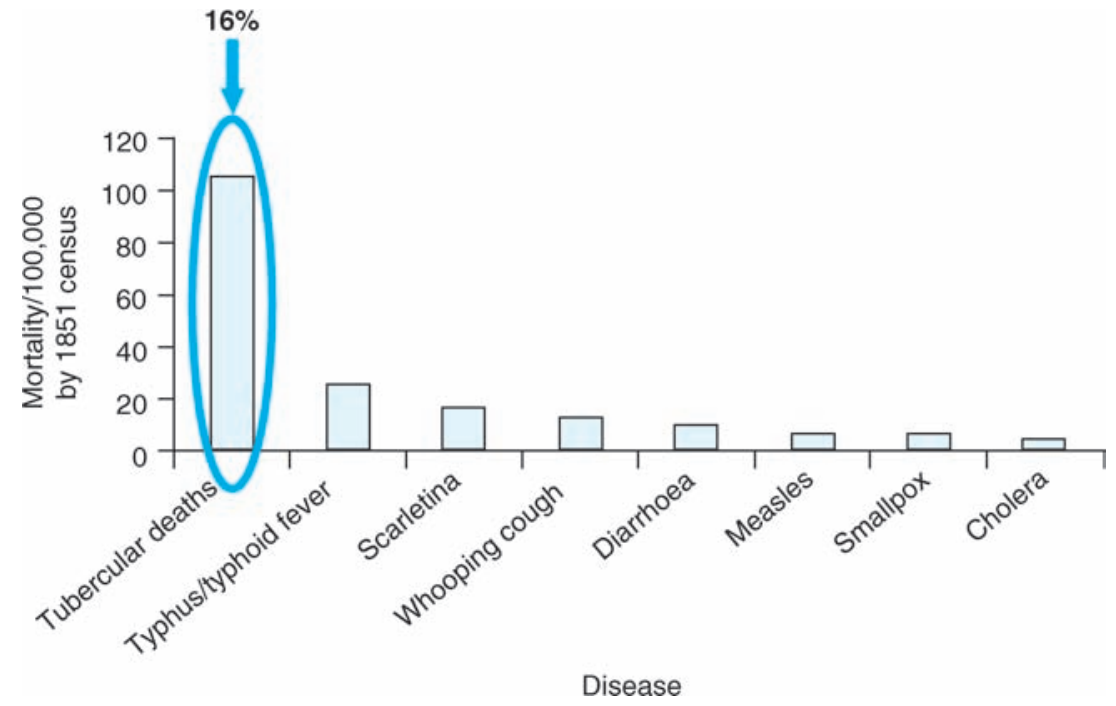


total of 30 million individuals tuberculin tested and 14 million given BCG vaccination. This vaccination programme was extended by the WHO in 1974, aiming for 70-95\% BCG coverage. ${ }^{23}$ However, the effectiveness of BCG in prevention of adult pulmonary disease is limited, ${ }^{26}$ although it protects against severe childhood infection. ${ }^{27}$ This has resulted in a number of strategies to develop a more effective vaccine, ${ }^{26,28}$ but perhaps the most telling aspect is the sheer number of candidates and approaches, indicating that we are still some way from achieving this goal.

These attempts at control of TB are of particular significance in the development of public funding for medical research in the UK. In 1908, the Royal Commission into the Relations of Human and Animal Tuberculosis, 1901-11, recommended a permanent medical research body and this was included in the 1911 National Insurance Act of Provision for Sanatorium Benefit, introduced by David Lloyd George. ${ }^{29}$ One provision paid for with a penny per working person per year - was sanatorium treatment for cases of TB and for 'purposes of research'.
This created a national fund for medical research and amounted to $\mathfrak{E} 57,000$ a year - equivalent to nearly $\mathfrak{E} 4$ million today. This research was not restricted to TB when the forerunner of today's Medical Research Council (MRC) - the Medical Research Committee - was formed. It developed a national 'scheme of research' and decided to invest its resources in scientists including 'exceptional' researchers given a salary and pension so that they could devote their whole time to research. Furthermore, it recognised that 'efforts should also be made to retain for research young and talented investigators who would otherwise tend to drift into other lines. ${ }^{29}$ Debates continued around how resource should be allocated and in 1918 the idea that the MRC should make scientific decisions independently from government was first proposed by Lord Haldane's Committee - now known as the 'Haldane Principle'.

Although the operations of the Medical Research Committee are within the province of the Minister responsible for Health Insurance... the Minister relies ... upon the MRC to select the objects upon which they will spend their income, and to frame schemes for the efficient and economical performance of their work. ${ }^{29}$
Fig 2a, b. Mortality from tuberculosis per 100,000 of the population by country (a) and major city (b). Data from Ref 22 .
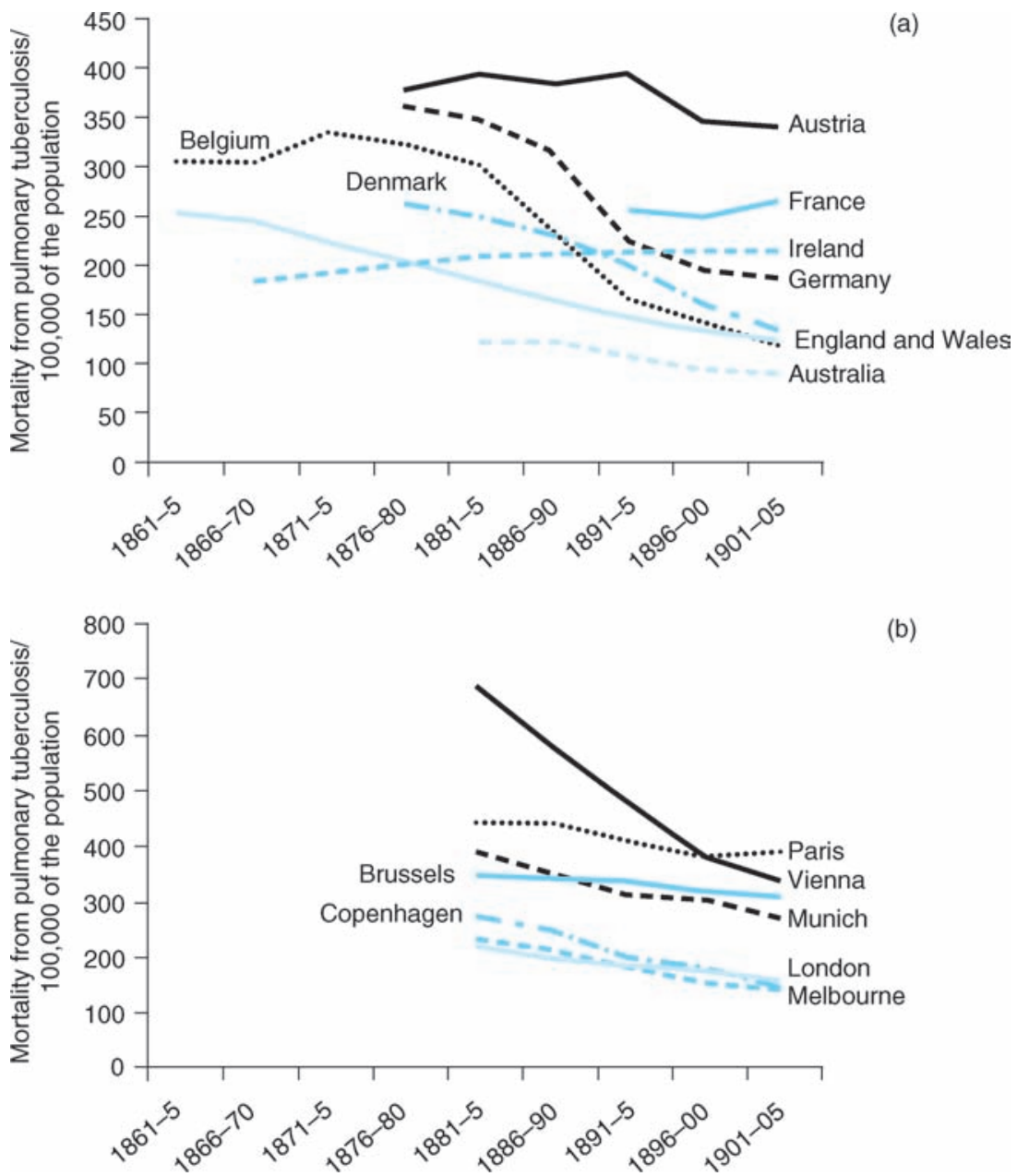
While the MRC made a major impact on many disorders, its impact on TB research was delayed, but important. In 1946, the MRC undertook the first clinical trial with randomised entry using streptomycin. ${ }^{30}$ This was ethically acceptable because of the small amount of streptomycin available and this could be compared with the best available care which was bed rest alone. The MRC established the MRC Tuberculosis Research Units whose major impact has been summarised in Box $1 .^{31}$

However, the true worth of this background clinical research is coming to the forefront in view of the changing nature of the problem TB poses today. In fact many of the current issues with TB were identified in these studies. Three major factors have emerged, which have changed the approach that we need to adopt:

1 The emergence of HIV with consequent increased susceptibility to TB. This is an additional burden particularly in high HIV-prevalence countries where the joint burden is overwhelming. The number of new worldwide cases of TB is estimated at 9.2 million and 1.7 million die of the disease each year. ${ }^{32}$ TB and HIV are the leading independent causes

Box 1. Summary of the major impact of the Medical Research Committee's Tuberculosis Research Units.

1946: The initial trial assessing the value of the addition of streptomycin to bed rest.

1948: The demonstration that the emergence of bacterial resistance to either streptomycin or p-aminosalicylic acid (PAS) alone was greatly decreased when combined treatment was given with both drugs.

1952-5: Exploration of treatment with isoniazid alone and in combination with PAS or streptomycin.

1958-67: The search for affordable regimens for developing countries that led to the substitution of thiacetazone for PAS.

1959: The demonstration that chemotherapy given at home was as effective as when given in a sanatorium and did not lead to any increase in the rate of infection in family contacts.

1958 onwards: Initiation of the policy of full supervision of chemotherapy (directly observed treatment (DOT)) and its later implementation in Hong Kong and Madras.

1961 onwards: Exploration of intermittent regimens of chemotherapy to assist implementation of full supervision.

1970: The first demonstration that inclusion of rifampicin or pyrazinamide in a regimen of streptomycin and isoniazid substantially reduced the subsequent relapse rate.

1972-4: Demonstration that the period of treatment could be shortened to six months by the inclusion of rifampicin and pyrazinamide in the regimen.

1976: Delineation of modern short-course chemotherapy regimens by showing that the sterilising activity of pyrazinamide was confined to the first two months of treatment during the intensive phase, whereas the sterilising activity of rifampicin persisted throughout the continuation phase.

1977 onwards: Demonstration of the value of intermittency in short-course regimens, particularly that three times weekly treatment throughout was as effective as, and less toxic and expensive than, daily regimens. of death with 12-14 million people co-infected with these agents. ${ }^{33}$

2 In the 1990s, the emergence of multidrug-resistant TB (MDR-TB) was recognised, defined as resistance to at least two first-line drugs used in the treatment of TB. This problem was predicted in the earlier MRC studies ${ }^{31}$ and it may explain the initially observed therapeutic effect of streptomycin alone in $\mathrm{TB}$ meningitis where the number of infecting organisms is low. ${ }^{24}$ The underlying mycobacterial mechanisms of resistance are numerous ${ }^{34}$ and therefore it was little surprise when the condition was first recognised in communities where there was poor compliance with a protracted course of medication associated with a high mycobacterial burden. Initial detection was rapidly followed by discovery of an alarming rate in developing countries and Eastern Europe where there may be HIV co-infection, intermittent access to and poor quality of drugs. However, the scale of the problem is that in their last survey the WHO estimates that 5.3\% (95\% CIs, 3.9-6.6) of all global isolates are MDR. This masks a considerable difference between countries; for example, up to $35 \%$ of cases of TB were MDR-TB in the former Soviet Union. ${ }^{35}$ It is also widespread in surveys in China. The reported trends are also worrying, in that while Baltic countries seem to be bringing this under control, in Russia the proportion of MDR-TB continues to rise..$^{35,36}$

3 This difficult situation is made even worse by the emergence of 'extensively drug-resistant tuberculosis' (XDR-TB). This is defined as $\mathrm{M}$ tuberculosis, resistant to at least rifampicin and isoniazid, any fluroquinolone and at least one of three injectable second-line drugs. ${ }^{37}$ The WHO surveys recognised that approximately $2 \%$ of MDR-TB isolates were XDR-TB by resistance to three of six classes of second-line drugs ${ }^{37}$ and 49 countries have reported the presence of the organism. ${ }^{38}$ This is an underestimate of the potential problem for three reasons:

- The coverage of these surveys is patchy, particularly in Africa where the highest prevalence and incidence of TB infection is found. The capacity to detect XDR-TB is further limited by a very small number of laboratories, especially in Africa, hence the possibility of a significant underestimate.

- There is major variation of the fraction of XDR-TB in different countries; the rates are particularly high in the former Soviet Union where 10\% of all MDR-TB is XDR; the variation is from $4 \%$ in Armenia to $23.7 \%$ in Estonia.

- There is accumulating evidence of community-spread and primary infection with MDR- and XDR-TB. This was recognised in a study in Kwazulu Natal among HIVinfected subjects. ${ }^{39}$ The study examined material from HIV-infected patients with culture positive TB, who later developed MDR- or XDR-TB. The accepted explanation was an assumption of failed or poorly compliant therapy. However, in 17 subjects, genotyping of the initial isolate prior to initiation of therapy during both episodes indicated that they were the result of re-infection rather 
than resistance developing in the initial strain. Nosocomial infection was unlikely as there was substantial variability making a point source outbreak unlikely. ${ }^{40}$ This is supported by earlier reports which suggested exogenous re-infection. ${ }^{39}$ In this context, recent findings from Shanghai are equally worrying, if not definitive. Ming Zhao and colleagues studied 4,379 isolates of TB with drug susceptibility results from the 19,722 newly registered TB cases between 2004 and 2007..$^{41}$ They identified 247 (5.6\%) MDR cases and of the 175 cases with available isolates, 109 (62\%) were simple MDR, 55 pre-XDR (31\%) and 11 (7\%) XDR. Although HI status was not reported, $60 \%$ of MDR and $55 \%$ of XDR cases were newly diagnosed and had not received prior therapy.

Therefore in the context of prevention, we are faced with a new danger posed by MDR- and XDR-TB. However, primary prevention is not available; therefore prevention of the impact of this infection is based on reduction of transmission in the population by effective detection strategies and sterilising treatment of individual cases as well as general measures more reminiscent of the pre-antibiotic era of TB control. This has led to a major international effort through the WHO. ${ }^{32,35}$ The introduction of improved culture-based and other detection methods is ultimately critical to this effort ${ }^{26,42}$ as is the need for effective surveillance. ${ }^{32,36}$ Unfortunately, treatment regimes for MDR are complex with directly observed and long-term therapy and outcomes that remain poor often because of concomitant HIV infection. However, a recent meta-analysis ${ }^{43}$ suggests that outcomes can be improved. Important aspects were treatment duration of $>18$ months, directly observed therapy for the duration of treatment, but there was no significant difference between individualised as opposed to standardised therapies. This analysis could not address the other major difficulties that have to be faced, particularly in the resource-poor setting, notably patient support, follow-up, an organised delivery system and the need for long-term commitment by policy makers locally and internationally, as was underlined at the 62nd World Health Assembly, Beijing 2009, and the 2009 Pacific Health Summit devoted to this theme. ${ }^{44}$ Despite these efforts outcomes are still poor - mortality and/or treatment failure was $\sim 20 \%$ with a $12 \%$ default rate even in the context of clinical trials and co-infection with HIV greatly reduces successful treatment. ${ }^{43}$

For XDR-TB this is much worse: in Kwazulu Natal 52/54 XDR patients died with more than $70 \%$ dying within a month of sputum collection. ${ }^{45}$ However, the outcome is poor even in the absence of HIV infection: in South Korea, treatment failure was $44 \%$ compared with $27 \%$ of non-XDR patients; in Germany and Italy, a five-fold increased mortality was observed. ${ }^{37}$ The treatment regimes are long, complex and individualised, with high hospitalisation costs and protracted inpatient care, occasionally resorting to other measures reminiscent of what we thought was a bygone era, such as surgery. ${ }^{37}$

So in this instance, the major drawback is the need to develop better diagnostic and surveillance technologies and the need for new agents. ${ }^{26,46,47}$ Gains in treatment will certainly improve prevention of the spread of MDR and XDR but this must not deflect from the more difficult task that we still face of an effective immunisation to replace BCG to reduce the global burden of community-acquired TB. ${ }^{26}$ But as highlighted by the WHO, ${ }^{32}$ both the approach to global TB control, as well as the specific instances of the threat posed by MDR- and XDR-TB, will require an implementation programme that is supported by policy makers locally and internationally. The commitment to sufficient and long-term, consistent funding is key if a major impact is to be achieved. Therefore, our research efforts must embrace all these aspects. At one end of the spectrum we have to support the basic research, which is inherently difficult with mycobacteria, improve animal models of TB, develop new drugs ${ }^{47}$ and vaccines. ${ }^{26,32}$ Simultaneously clinical experimental approaches essential for proof of concept as well as trials not only to determine clinical effectiveness but also effectiveness for delivery systems and their suitability as well as sustainability are a pre-requisite for long-term success.

\section{Implementation of healthcare trials in resource-poor countries}

In last year's Harveian Oration, Professor Rawlins discussed the importance of clinical trials and their strengths and weaknesses in establishing an appropriate evidence base. ${ }^{48}$ The relatively simple historical studies conducted in the 1940s and 1950s have been superseded by developing novel methodologies and concern the implementation of appropriate therapies in the field setting. At the heart of any preventative measure, the value of the intervention has to be established. This will be increasingly important if a preventative intervention, for a common condition affecting resource-poor societies, is to become established in the future. An example of the problems such studies encounter is the Delivery of Antiretroviral Therapy (DART) study. This study was the largest clinical trial of antiretroviral therapy (ART) ever run in Africa for people with HIV infection. Its primary goal was to examine delivery of therapy rather than the actual therapy itself and the trial compared routine laboratory and clinical monitoring (LCM) for toxicity and efficacy with clinically-driven monitoring (CDM) (ie no CD4 counts were made available and routine toxicity test results were only available if severe toxicity noted, or when clinically requested).

The major result is that regular laboratory tests offered little additional clinical benefit to populations when compared with careful clinical monitoring. Over nearly five years, 90\% of participants in the LCM group compared with $87 \%$ in the CDM group were still alive. This small increased risk to the individual needs to be balanced against the increased costs and logistics of the LCM arm (around 27\% more expensive). Adding routine laboratory monitoring of antiretroviral therapy in DART, the incremental cost-effectiveness ratio, which relates the costs of the intervention to the survival benefit was estimated to be \$US 8,312, which is not cost effective in Uganda or Zimbabwe by WHO criteria of three times GDP. The cost of a CD4 count would need to drop to less than $\$ 4$ for this to be cost effective. 
Economic analyses in DART also suggest that additional life years lost through clinically-driven monitoring are very small compared with those lost by not providing ART. Implementing its findings would mean that more people with HIV in Africa could be treated for the same amount of money as is currently spent if laboratory tests are not routinely used to monitor the effects of antiretroviral therapy. This challenges local and global health policy makers and others who make funding decisions to provide antiretroviral therapy, as well as the affected community, to implement the findings. This last is the most difficult to achieve, as the received wisdom has been that antiretroviral therapy can be effective only with the accompanying laboratory tests, which will inevitably raise the suspicion that those in developing countries are being given second-class care.

While the specific findings are extremely important for HIV patients, there are several issues raised which will have to be considered before embarking on studies of implementation in a resource-poor setting:

- In total, 3,316 people took part in the DART trial, alongside 199 investigators from a myriad of clinical and non-clinical disciplines, 173 based in Africa. This raises questions of their career development and capacity building for participating investigators. These are important goals and trials such as this provide excellent opportunities but a major financial commitment in parallel to the main studies is required to reap these benefits.

- This study and many others have resulted in a large number of publications related to secondary endpoints and embedded studies taking advantage of the cohort established for the primary endpoint (to date 10 publications and 40 presentations). It remains essential that these do not overwhelm the primary goal but that where new and interesting data emerge, there is the opportunity to follow up. This is essential for maintaining morale and career development but often leads to frustration on the part of the investigators, when funders cannot support such work.

- MRC funding for DART was $£ 5.5$ million, but the MRC was one of three direct funders including Rockefeller and the Department for International Development, to a total direct cost of $£ 14.2$ million, which excludes provision of drugs as these were donated by the pharmaceutical industry. Studies such as this will increasingly require a multiplicity of funders who have to have a shared commitment to the study and ultimately its consequential effects. The sheer financial commitment will mean the need for increasing prioritisation by consortia of funders and this will inevitably raise major issues between very committed investigators whose proposals are not prioritised. Furthermore, most studies in preventative medicine will not involve a commercially relevant endpoint, so additional pressure will fall on the public and charity sectors. The end result is that, even with commitment by funders, progress will be slow and frustrating for all concerned - funders, investigators, policy makers and most importantly the patients and wider public alike. Therefore there will need to be extensive and open debate about wider implementation of some interventions, without the benefit of a 'gold standard' of evidence provided by a clinical trial in many instances.

- This study was scheduled for a primary endpoint after five years, yet to achieve primary endpoints in many future studies will require decades of follow-up as well as large populations in different geopolitical contexts to determine sustainability and applicability. An early example is the MRC Gambia Hepatitis study, which was started in 1986 with national hepatitis B (HBV) vaccination of $\sim 60,000$ infants over a four-year period compared with an equal number of nonvaccinated controls. The end point of the study was diagnosis of hepatocellular cancer and other chronic liver diseases after 40 years. This has required appropriate follow-up and the establishment of facilities to provide disease-specific surveillance in the field. While this study continues, ${ }^{49,50}$ and new methods of detection of cases and controls are being developed, it is extremely difficult to maintain the commitment from subjects, staff and particularly funders, especially if they can argue that the issue of value has already been established, albeit in entirely different clinical settings. ${ }^{51}$ Therefore, long-term commitment is key and only in the presence of such commitment can there be any expectation that investigators and subjects will engage to achieve the primary endpoints.

These issues will be encountered in specific interventions and in the progress to preventing common non-communicable diseases. Funders and policy makers will have to decide what evidence they require to initiate an intervention and how best to utilise the limited resource available to obtain it.

\section{Cervical cancer vaccines}

Cancer of the cervix remains the second most common cancer of women worldwide causing 260,000 deaths annually with $80 \%$ of cases in resource-poor countries in sub- Saharan Africa and South America. Dominantly, it remains a disease of the poorest sectors of society, and is the cancer with the strongest association with low socioeconomic status even in the poorest countries. ${ }^{52,53}$ In western countries screening by cervical cytology has reduced the incidence of the disease and modification of screening modalities may further enhance its clinical and cost effectiveness. ${ }^{54}$ The association between human papillomavirus (HPV) and cervical cancer was established by Harald zur Hausen, for which he received the 2008 Nobel Prize, ${ }^{55}$ and has been confirmed in many studies. ${ }^{56}$ Epidemiological studies by the International Agency for Research on Cancer (IARC) established that most cervical cancer in the global context was associated with HPV types 16 and 18.57 HPVs are a large family of double stranded, non-enveloped DNA viruses that are species specific with distinct tropisms linked to their genomic subtype. Genital infection with HPVs, especially oncogenic types, is extremely common and often occurs shortly after onset of sexual activity. ${ }^{58}$ This epidemiology is supported by a large body of basic research which established the oncogenic potential of 
two non-structural proteins E6 and E7 in HPVs associated with malignant transformation. ${ }^{59,60}$ These proteins interfere with $\mathrm{p} 53$ and $\mathrm{Rb}$ in the cell allowing disordered regulation of growth. If the virus persists, additional mutations may occur which in turn transform the cells through the characteristic changes and stages observed by cervical cytology, histology and ultimately clinical invasiveness. ${ }^{56}$ This process is dependent on virus persistence, additional co-factors and associated risk factors but also a prolonged time-frame. ${ }^{55,56,61}$ The length of time and frequency of natural regression at all stages of pre-invasiveness accounts for the success of cervical screening and the success of clinical management of early stage invasive disease. ${ }^{61,62}$

Two approaches to utilise the antigenicity of virus proteins in treatment and prevention have been adopted. We had a longstanding interest in the role of cellmediated immunity in human persistent virus infection. ${ }^{63}$ E6 and E7 were key proteins that have to be continually expressed to maintain the transformed state; therefore it was possible that CD8 + cytotoxic T cells could be immunotherapeutic in patients with established malignancy. To this end, a vaccinia virus vector was prepared with changes introduced by site directed mutagenesis into the $\mathrm{Rb}$ binding site. ${ }^{64}$ This construct was used to vaccinate nine subjects with late stage cervical cancer and CD8 + cytotoxic T cells specific for the viral proteins were induced (Fig 3). ${ }^{65}$ These cells were also detected in patients with cervical cancer and pre-invasive disease and localised to site of disease. ${ }^{66,67}$ However, cervical cancer cells were able to evade this immune response ${ }^{68,69}$ and this was associated with mutations in cancer cells that interfered with $\mathrm{MHC}$ class I presentation. ${ }^{70}$ It is probable that E6- and E7-specific MHC class I restricted cytotoxic $\mathrm{T}$ cells are generated in response to genital HPV infection and these cells may clear infection. The high frequency of mutations in the MHC class I antigen presenting pathway also suggests that this is an important mechanism for limiting spread as evasion of this immune response is an important accompaniment for effective invasion. Therefore this approach to immunotherapy will require not only generation of these effector cells but also adjuventicity in the preparation to alter the local ability of the target cells to present antigen.

While immunotherapy would be a useful adjunct to available treatments, primary prevention of disease has to be an ultimate goal. Two groups were able independently to generate 'virus-like particles' (VLP) which were shown to be immunogenic and protective in animal models. ${ }^{71,72}$ These particles consisting of the structural proteins that make up the virion were formulated and developed into two vaccine preparations: a quadrivalent vaccine incorporating L1 and L2 proteins from HPV 16 and 18 but also from two common types associated with genital warts HPV 6 and 11 (Gardasil, Merck) and a bivalent HPV 16 and 18 product (Cervarix, GSK). The results of the first human studies were dramatic. ${ }^{73}$ There was strong immunogenicity (vaccine recipients mean 1,510 antibody units vs $<6$ in the control group $(n=619$ vs 631) and primary analysis of virus persistence for $>6$ months was $0 / 100$ women years vs $3.8 / 100$ women years, equating to all 41 'cases' falling in the control group. Although the follow-up period was short, there were no significant adverse events noted. These studies have been followed up with longer-term studies and the early results continue to show sustained benefit. ${ }^{74-77}$ Interestingly, some studies suggest that there may be cross-reactive protection to closely related HPVs ${ }^{75}$ but, as expected these vaccines have little effect on those already infected. These exciting results have brought about considerable change in policy and in the UK national vaccination is being implemented. However, several issues remain to be resolved:

- vaccination is proposed at the age of 11 years - what would be the value of catch-up vaccination at older ages?

- access to screening will need to continue but should this programme be amended?

- should men be vaccinated especially if this vaccine could protect against anal cancer?

- what should be the degree of coverage in the population and is there going to be a requirement for revaccination?

- what are the long-term safety implications?

- is it cost effective?

These are independently significant issues in their own right but are also dependent on each other. Furthermore, it will fuel the debate about acceptability because the very nature of this immunisation as a protection against a sexually transmitted disease is highly emotive and raises opposition of itself in sectors of society. Offering vaccination to other age groups is dependent on the onset of sexual activity and likelihood of prior infection. Recent studies from Bolivia have shown that the vaccine is equally effective in inducing immunity in women aged 25-45 years, who had not previously been infected. ${ }^{78,79}$

Most studies of cost effectiveness have to rely on assumptions in defining these key parameters. It is clear that there will be a reduction in cervical cancer if $90 \%$ of all girls aged 11 to 12 are vaccinated, a target that will have to be achieved for $30+$ consecutive years and assuming that vaccination provides life-long protection. ${ }^{80}$ While current information supports long-lived immunity, ${ }^{74}$ this will need to be constantly monitored by cervical screening to ensure that we do not have a population of women who may become susceptible later in life in the presence of antibody but at non-protective levels. Clearly revaccination will be an option but this will add considerably to the cost burden. ${ }^{81}$ Nevertheless despite these reservations the modelling and available data suggest that this is a vaccine suitable to a national programme.

This exposes the other questions that need to be addressed. Firstly, should men be vaccinated both as a 'vector' of the disease and in prevention of other HPV associated lesions. The latter question remains open but most modelling indicates that if the primary endpoint is prevention of cervical cancer then $80 \%$ coverage of girls will be required and the additional impact of vaccinating boys is not likely to be cost effective. ${ }^{82}$

Secondly, there is no saving to be made by a reduction in screening. There are a multiplicity of reasons for this but in the forefront is the unknown duration of protection, the limited number of oncogenic HPV types included in the vaccine, cover for those not vaccinated and the unknown level of herd immunity 
that may be generated. Is the current screening programme optimal to address these issues in the future? The cervical cancer screening programme has been a major success story in Western countries and has undoubtedly reduced the incidence of and mortality from the disease ${ }^{83,84}$ Now the condition is mostly encountered in those who do not attend screening with recall. Recent studies underline the importance of using both HPV and cytologic assessment:

- Castle and colleagues found, in a study based in Costa Rica, that $20 \%$ of women with one-year persistence of HPV and $40 \%$ with HPV-16 persistence will develop cervical precancer in the subsequent $3-5$ years. ${ }^{85}$

- Sasieni and colleagues found that cervical cytology is effective between ages 35-64 but less so between 25-34 and not all between $20-24 .{ }^{86}$

- The TOMBOLA Group, studying the optimum management of low-grade lesions detected at cytology, found that immediate referral for colposcopy had no benefit over cytological surveillance; ${ }^{87}$ biopsy and large loop excision of the transformation zone were equally effective but the latter over treated. ${ }^{88}$ Furthermore if these three approaches are compared for cost effectiveness then there is no significant difference. ${ }^{89}$

- HPV testing may also allow for more effective stratification of disease. ${ }^{90}$

So what will become the optimum screening programme to support HPV vaccination? Currently, most data would support HPV testing at extended intervals to replace cytology as a primary screening modality, adding cytology to stratify those at highest risk. ${ }^{91}$ However, this transition will in itself be costly in terms of education, clinical practice (especially to avoid overtreatment) and acceptance of changed guidelines. But ultimately patient acceptability will be a challenge because of the increased interval between screening (the 'worried well') and consequent change in clinical practice. There will be consider- able difficulty in getting patients to accept that recent-onset HPV is benign and may not require any intervention and surveillance may become more important than immediate intervention - both may be unacceptable to many and this has to be anticipated and countered.

Thirdly, the issue of acceptability has to extend not just to changes in cervical screening but whether this vaccine will be accepted by the population at risk and their parents, as well as by society at large. ${ }^{92-94}$ Several themes emerge:

- What is the individual cost:benefit ratio as interpreted by the patient, parent and individual clinician, bearing in mind the small risks for individuals if screening procedures are properly utilised? This will depend on a better understanding of risks.

- The major side effects are currently monitored by the US Vaccine Adverse Events Reporting System (VAERS) and include anaphylaxis, Guillain-Barre syndrome, transverse myelitis, pancreatitis and deep venous thrombosis. Most of these events were not greater than background rates compared with other vaccines but this is a voluntary reporting system and it cannot carry the same weight as follow-up in controlled trials. ${ }^{93}$ How results are presented and considered in the individual patient encounter is critical.

- Equally worrying is the perception that the agencies and professional associations in the USA that have been promoting vaccination were supported by industry, ${ }^{92}$ even in advance of studies showing clinical endpoint benefit. ${ }^{94,95}$

Whether these suggestions reflect bias is less important than raising that perception. Independent advice and a careful approach to understanding and responding where there are concerns - real or imagined - are essential. If not then yet again we will have failed to learn from the experience of other vaccines ranging from smallpox to MMR. Ultimately the issue now is not one of technical achievement but of acceptability to ensure effective adoption of what is likely to be a defining intervention in this disease.

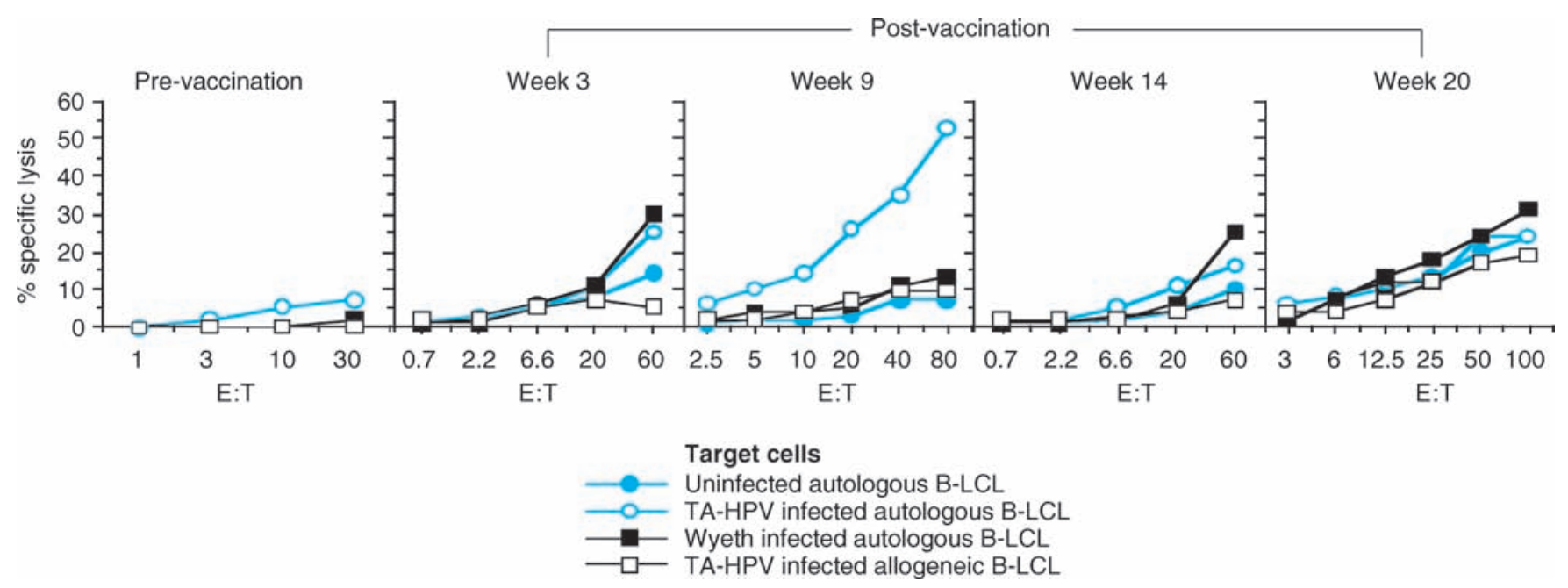

Fig 3. The generation of HPV-specific, CD8+ cytotoxic T cells after vaccination with a vaccinia recombinant expressing modified HPV type 16 and 18, E6 and E7. CITATION Bor96 \ 2057. ${ }^{65} \mathrm{E}=$ effector; $\mathrm{T}=$ target. 
All of these issues are substantial, particularly in high-income countries, yet the full benefit will only be achieved globally if the vaccine is widely adopted in resource-poor settings. Is this realistic? In most developing countries, studies of prevalent HPV in tumour samples suggest that HPV 16 and 18 still dominate and even the current limited formulation could make a significant impact on the prevalence and incidence of the disease. ${ }^{56,57}$ It is widely reported that the prevalence of HPV infection with other oncogenic HPV types is more widespread and diverse than in western countries (eg The Gambia ${ }^{96}$ ). Many current and future intervention studies will be performed in this setting but the question remains as to the feasibility of implementation in these countries. Issues include:

- Applicability. This will be addressed by current studies but the key issue is whether this represents the best use of resources in the many calls on limited resource? Secondly, is this the only effective approach to cervical cancer control?

- Cost. This relates not just to the cost of the vaccine but establishing the whole system cost of vaccine delivery especially to older subjects, and the clinical care required for effective implementation and care of detected cases.

- Access to screening with effective follow-up.

- Acceptability of this vaccine by the community. Education and an understanding by the community of the nature of cervical cancer are essential. This must go alongside the WHO Millennium Development Goal of education, especially for girls in these societies.

- Where will this sit as a priority where there are limited resources in a country?

- Does the international community feel strongly enough about the issue to provide the funding and commitment required to tackle cervical cancer?

It remains difficult to imagine that the full range of services needed to manage disease detected by screening can be readily developed in all countries but some will consider this a realisable goal. The very fact that screening may move away from cytology may be a significant factor for many. The recent study of HPV screening in India by the IARC has raised significant issues. ${ }^{97}$ In this study, cluster randomisation by village was used to trial three screening modalities: HPV testing, cytology and visual inspection with acetic acid. The overall result was that only HPV testing was associated with a significant reduction in the numbers of advanced cancers and mortality from cervical cancer. This will need to be considered alongside, and by some, as an alternative to vaccination. Trials to define optimum prevention, surveillance, clinical care and cost-effectiveness strategies in this setting will be essential but their nature and complexity will be akin to the DART trial and require the long-term commitment of funders and investigators.

\section{The problem of non-communicable diseases}

Cervical cancer may be considered to be the meeting point of problems posed by infectious and non-communicable diseases, yet as a prevention issue it is a chronic consequence of a sexually transmitted infectious disease. The real burden of disease looming in the developing world is with 'diseases of affluence'. While many resource-poor countries are wrestling with the issues of infectious disease, this problem is approaching them at an ever-accelerating rate. It was predictable and predicted in this very lecture by Richard Doll..$^{98}$

Non-communicable diseases now account for $60 \%$ of all deaths worldwide and in contrast to popular belief, $80 \%$ of deaths from these conditions occur in the developing world. ${ }^{99}$ The major causes are cardiovascular disease, cancer (especially smoking related cancers), chronic respiratory disease and diabetes. Even more worrying than the statistics in terms of total mortality, is the observation that $44 \%$ of all preventable mortality resides in the developing world, which is approximately twice the number of preventable deaths from communicable diseases. ${ }^{99}$

A Delphi study was undertaken, which identified six major goals, 29 grand challenges and 39 substantive research questions. ${ }^{99}$ The major challenges include:

1 Raising public awareness of the problem.

2 Adaptation of the economic, legal and environmental policies of all governments to take into account these issues.

3 Direct modification of significant risk factors.

4 Engagement with local communities to understand the impact locally as well as greater involvement of the business and commercial sectors, which ultimately will benefit from improved health gain.

5 Recognition of the importance of dealing with poverty and the consequences of unplanned urbanisation.

6 Re-orientation of health systems from dealing with a dominantly communicable diseases orientation to develop the capacity to build appropriate chronic care models.

These issues are challenges for the global community and cannot be left to local intervention alone. To this end, heads of publicly-funded biomedical research organisations put together a framework to engage partners who would be willing to commit resources to the challenges posed by non-communicable diseases (Fig 4). The current committed participants include the MRC, National Institutes of Health, Canadian Institute of Health Research, National Health and Medical Research Council of Australia, Chinese Academy of Medical Sciences and the Indian Council for Medical Research. ${ }^{100}$ The partners will commit resources to enable research focussing on prevention and implementation of intervention leading to health benefit. The specifics will be developed with the research community (November 2009) to define research priorities, although these are likely to include an initial focus on dietary and respiratory diseases. Further themes, such as the importance and impact of psychiatric illness, may follow. This alliance will be independent of any individual funder and will seek to work with others who share and recognise the significance of these goals in the wider importance of developing interventions that can truly prevent the major global scourges that resource-poor countries will face in the near future.

This enterprise poses new challenges for preventative medicine and a focus on implementation rather than new discovery 
Fig 4. The establishment of the Global Alliance for Non-Communicable Diseases. NHMRC $=$ National Health and Medical Research Council of Australia; MRC $=$ Medical Research Council; $\mathrm{ClHR}=$ Canadian Institute of Health Research; $\mathrm{NIH}=$ National Institutes of Health; $\mathrm{MoH}=$ Ministry of Health/Chinese Academy of Medical Sciences.

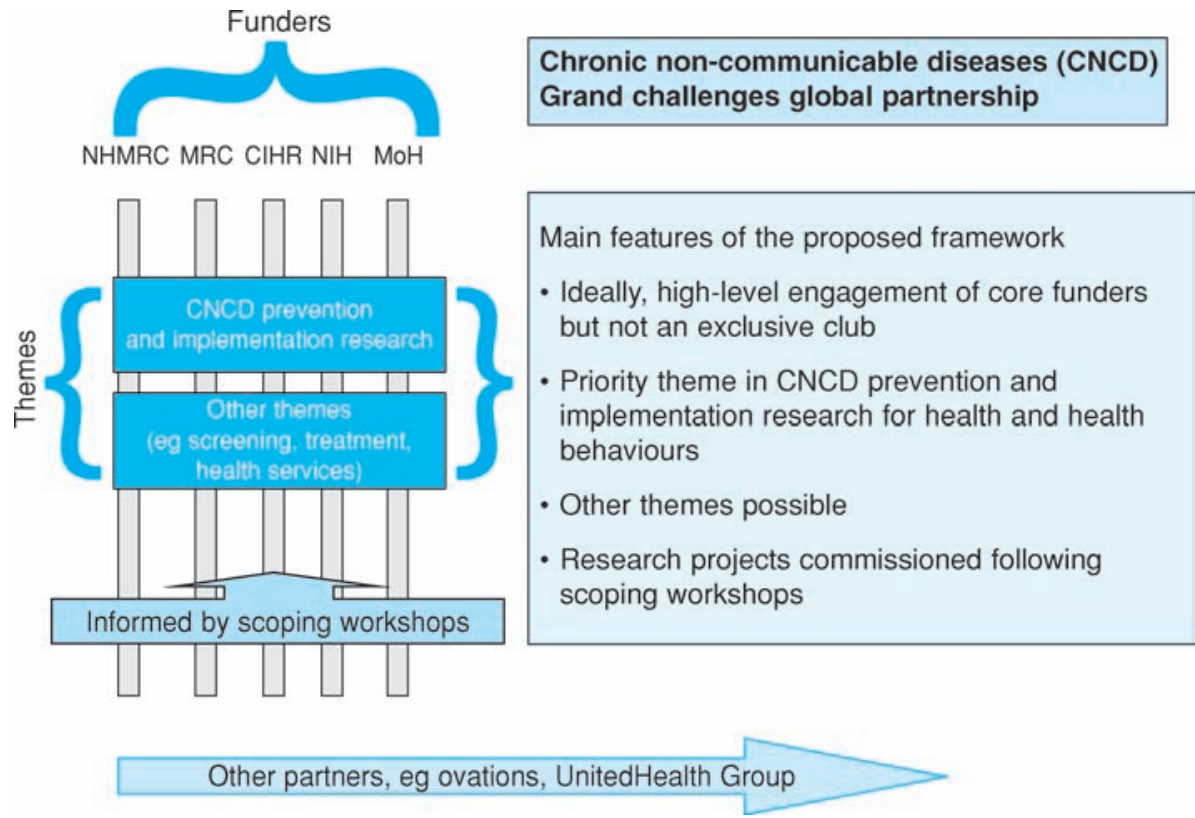

is key if any impact is to be made on this burgeoning global problem.

\section{Conclusions}

The adage 'prevention is better than cure' is as apposite today as it has always been. Sir Richard Doll was over optimistic in his Harveian Oration ${ }^{98}$ about our capacity to influence and change behaviour to deliver the improvement in health that the evidence base he helped develop should drive us towards. This is not an excuse to redirect all science to translation and implementation. That would be short-sighted and unproductive. It is a continued commitment to basic science, in all its guises and disciplines, which provides the foundation and technology required to initiate and develop change central to successful prevention. As I hope that I have shown, it is essential that this commitment extends to continued support for clinical experimentation, both for discovery as well as effective translation for clinical benefit. This must include, for the foreseeable future, the need for animal models as a major aspect of understanding pathophysiology and pre-clinical trials. Evaluation of intervention in all historical and current examples is essential to ensure that the proposed change is optimal for their desired goal. Evaluation is not optional but integral to every large-scale prevention proposed. Although, as I have indicated, the nature of evaluation varies with the proposed intervention. These evaluations are going to be expensive and difficult especially if clinical trials are utilised. Commitment to all these goals must be long term and in part protected from the vagaries of policy makers by giving them a greater understanding of why this is absolutely necessary to support policy decisions. And last but not least, as in every example I have chosen, the importance of ensuring widespread understanding and public support is critical to any preventative measure we may seek to introduce. This requires us to recognise the vagaries of human behaviour in relation to healthcare benefits, which are not new and have always been and will continue to be present, in any democratic society. If we espouse the goals of prevention, as did the physicians in relation to smallpox vaccination in the 19th century, we must not fall into the traps that they did and maybe we continue to do today. That is not only costly in terms of effort and finance, but more importantly impedes or blocks potential health benefits to the wider population. This means recognising that there is a strong research base in other academic disciplines such as social sciences, economics and business studies in terms of optimum marketing. Above all, in embarking on this we have to remember that we have to retain scientific objectivity and not to proselytise or engage in propaganda but to reason, debate and listen to issues that are raised. It is essential to recognise that there will never be unanimous agreement with any preventative intervention but we have to ensure that we carry the majority and empower their voice to be heard above the often raucous views expressed by committed opponents.

Prevention is better than cure and we have the opportunity within our grasp to make a major difference in some of the scourges that afflict mankind today. It would be very easy to restate the vision, but delivery of the vision has been, and will continue to be, a far more complex and challenging operation. Without delivery the visions themselves are pipe dreams and would represent a failure on our part to deliver real health benefit.

References are available in the full version of the Harveian Oration at www.rcplondon.ac.uk/pubs/brochure.aspx? $\mathrm{e}=292$

Address for correspondence: Professor Sir L Borysiewicz, Medical Research Council, 20 Park Crescent, London W1B $1 \mathrm{AL}$. 